\title{
Valoraciones acerca de las prácticas profesionalizantes del sector informática del nivel superior técnico
}

\author{
Assessments of Professional Practices in IT Professional \\ Technician's Training
}

DOI: https://doi.org/10.32870/dse.v0i22.703

Ana María D'Andrea*

María Paula Buontempo**

Federico Butti***

\begin{abstract}
Resumen
Este artículo se orienta a analizar las valoraciones acerca de las prácticas profesionalizantes de los diferentes actores vinculados a las mismas (técnicos, directivos, docentes, estudiantes, egresados, empresarios) en la Tecnicatura Superior en Soporte de Infraestructura de Tecnología de la Información de la provincia de Corrientes (Argentina). Las prácticas profesionalizantes constituyen un instrumento de la política educativa. En este trabajo se entiende la política educativa como el resultado de un proceso de construcción social configurado por un campo en disputa entre distintos actores o grupos que intervienen con diferentes recursos, intereses y valoraciones. El estudio es de carácter descriptivo. Se trabaja con una muestra intencional de tres carreras. Se utilizan cuestionarios con los estudiantes y entrevistas en profundidad con los distintos actores que intervienen en este dispositivo de formación. Como resultado se observa que las prácticas profesionalizantes, en general, son valoradas positivamente, aunque con diversos matices producto de los intereses y posicionamientos de los distintos actores. Las limitaciones se refieren a condiciones contextuales, a falta de recursos materiales - principalmente conectividad- $y$ a que algunas actividades que se realizan no corresponden al perfil profesional. No obstante, sobre las dificultades predominan las valoraciones positivas, coincidiendo en el potencial formativo que detentan.
\end{abstract}

Palabras clave: educación y trabajo - enseñanza técnica - enseñanza superior - tecnología de la comunicación - valoración (Tesauro de la UNESCO).

\section{Abstract}

This article aims to analyse assessments of IT graduates' professional practices made by different actors linked to them (technicians, managers, teachers, students, graduates, businessmen) in the Professional Technician in Information Technology Infrastructure Support program of the province of Corrientes (Argenti-

\footnotetext{
* Doctora en Antropología Social. Profesora en Ciencias de la Educación. Técnica-docente del Ministerio de Educación de la Provincia de Corrientes. Docente-investigadora de la Universidad Nacional del Nordeste. Argentina. anadandrea@gmail.com

** Doctora en Antropología Social. Técnica-docente de la Dirección de Planeamiento e Investigación Educativa del Ministerio de Educación de la Provincia de Corrientes. Docente-investigadora de la Universidad Nacional del Nordeste. Argentina. buontempop@gmail.com

*** Licenciado en Psicología. Especialista en Docencia Universitaria. Docente-investigador de la Universidad Nacional del Nordeste. Argentina. fbutti@hotmail.com
} 
na). Professional Practices constitute an instrument of educational policy. In this paper, educational policy is understood as the result of a social construction process shaped by a field in dispute among different actors or groups that intervene with different resources, interests and assessments. This descriptive study relied on an intentional sample of three majors. Questionnaires with students and in-depth interviews with the different actors involved in this training program were used. As a result, it was observed that professional practices are generally valued positively, albeit with different nuances resulting from the interests and positions of the different actors. The limitations refer to contextual conditions, the absence of material resources - mainly connectivity - and the fact that some of the activities conducted do not correspond to the professional profile. Despite the difficulties, however, positive assessments are predominant, coinciding with the training potential these professional practices have.

Keywords: education and work - technical education - higher education - communication technology assessment (UNESCO Thesaurus).

\section{Introducción}

\section{El problema}

La posibilidad de que los jóvenes estén desempleados es tres veces mayor que la de los adultos, con una tasa de desempleo mundial juvenil de 13 puntos porcentuales en 2017. Sumado a esto, las desigualdades que enfrentan los jóvenes se articulan con las desigualdades socioeconómicas, de género, étnico-raciales y territoriales, potenciándose y encadenándose a lo largo del ciclo de vida (ONU, 2018).

Según datos de la Comisión Económica para América Latina y el Caribe (CEPAL), en América Latina y el Caribe la mitad de los jóvenes de 20 a 24 años no habían concluido la educación secundaria a principios de 2019 , sus tasas de desempleo eran de $13 \%$, y $24.5 \%$ no estudiaban ni trabajaban en forma remunerada (CEPAL, 2019).

Respecto al sector informático, se plantea una situación paradojal. A pesar de que tenemos muchos jóvenes desempleados, este sector tiene dificultades para encontrar personas con habilidades tecnológicas adecuadas. Según un estudio de la Comisión Europea, se estima que habrá ochenta mil vacantes en el sector informática en 2020 (Sistema de Información de Tendencias Educativas de América Latina - SITEAL, 2019).

La modalidad del sistema educativo argentino que atiende la relación educación y trabajo es la de educación técnico-profesional. Durante la última década del siglo XX, el sistema educativo argentino atravesó por varias trasformaciones. Como consecuencia, la educación técnico-profesional fue relegada, desfinanciada y desmembrada (Gallart et al., 2003).

A partir del siglo XXI, un conjunto de políticas y regulaciones diseñadas e implementadas especialmente por los ministerios de Educación y de Trabajo, Empleo y Seguridad Social cambiaron de manera sustancial el panorama de desinversión y abandono de la modalidad técnico-profesional y contribuyeron a una nueva etapa de institucionalización. Uno de los hi- 
tos más importantes fue la sanción, en 2005, de la Ley 26.058 de Educación Técnico-Profesional (LETP), que propuso la reorganización y promoción del sistema educativo técnico argentino (Jacinto, 2015). La nueva LETP introdujo una innovación importante en materia curricular: las Prácticas Profesionalizantes (PP) como campo formativo obligatorio en la educación técnicoprofesional.

Las PP son un instrumento de la política educativa, es decir, "un dispositivo al mismo tiempo técnico y social que organiza relaciones sociales específicas entre el poder público y sus destinatarios en función de representaciones y de significados de los que es portador" (Lascoumes, Le Galès, 2014: 113). Así, aparte de su dimensión de objeto, un instrumento es también un acervo de traducciones e interpretaciones que varían en el espacio, en el tiempo y según los actores (Fontaine, 2015), con lo que queremos remarcar el carácter problemático de toda implementación en la arena de la política pública, donde se disputan sentidos y acciones (Dragone, Quinzani, 2018).

\section{Las prácticas profesionalizantes}

El documento de PP del Instituto Nacional de Educación Tecnológica (INET, 2007) y la Resolución $N^{\circ} 47 / 08$ del Consejo Federal de Educación (CFE) son los instrumentos principales a través de los cuales el Estado nacional y las jurisdicciones establecieron los límites del concepto de PP y sus lineamientos básicos. La provincia de Corrientes emitió una normativa que homologó aquellas con la Resolución № 612/11, pero solamente se refirió a la Educación Secundaria Técnica.

Así, las PP pueden ser de diferentes tipos y presentar diversos formatos para su organización (estudio de casos, trabajo de campo, modelización, resolución de situaciones-problema, elaboración de hipótesis de trabajo, simulaciones, actividades experimentales, entre otros) y llevarse a cabo en distintos entornos (laboratorios, talleres, unidades productivas, entre otros), tienen carácter institucional y son planificadas, programadas y supervisadas por los equipos docentes. Su objetivo principal es poner en práctica saberes profesionales sobre procesos socioproductivos de bienes y servicios relacionados con el futuro entorno de trabajo del estudiante. Asimismo, pretenden introducir y familiarizar a los estudiantes en los procesos y el ejercicio profesional vigentes (Ministerio de Educación de la Nación - MEN, INET, 2007).

Do Pico (2013) argumenta que una PP no es igual a cualquier práctica. En primer lugar, para ser profesionalizante debe estar alineada con el perfil profesional del egresado, produciendo una vinculación significativa entre la formación que da la institución educativa y los requerimientos emergentes de los sectores científicos, tecnológicos y socioproductivos. En segundo lugar, es importante marcar la diferencia entre las PP y el resto de la formación práctica de la educación técnica, en tanto las primeras están centradas en la vinculación con el mundo del trabajo y con actores del proceso productivo local (MEN, CFE, 2008). De ese modo, uno de los principales desafíos que se presentan, a las instituciones de educación técnica es la vinculación 
más estrecha con el afuera y con actores que no pertenecen al sistema educativo (Do Pico, 2013). En esa perspectiva, es necesario reconocer que las representaciones sociales, las expectativas y los intereses de los diversos sectores involucrados son diferentes. Las distintas lógicas que participan en estas relaciones, las cuales pertenecen a esferas muy distintas, tanto por sus contenidos como por sus actores, configuran un espacio de interacciones, compromisos y responsabilidades no exento de conflictos y necesarias negociaciones.

Con este marco, el artículo se orienta a analizar las valoraciones acerca de las PP por parte de los diferentes actores implicados en las mismas (técnicos, directivos, docentes, estudiantes, egresados, empresarios) en la Tecnicatura Superior en Soporte de Infraestructura de Tecnología de la Información de la provincia de Corrientes (Argentina).

Este trabajo forma parte de un estudio mayor, financiado por el Fondo Nacional de Investigaciones de Educación Técnico-Profesional (FoNIETP) del INET del Ministerio de Educación, Cultura, Ciencia y Tecnología de la Argentina.

\section{Valoraciones de los distintos actores respecto a la relación educación-trabajo} y la implementación de las prácticas profesionalizantes

Pacenza y Echeverría (2013) realizaron una revisión de la producción académica referida a los sentidos que los jóvenes atribuyen a la educación y el trabajo, y encontraron que las principales temáticas se refieren a la relación que tienen los jóvenes con el estudio y el trabajo en distintos sectores socioeconómicos, las expectativas de inserción laboral, los beneficios y perjuicios que los actores perciben que les genera trabajar y estudiar simultáneamente y las motivaciones que tienen para hacerlo; asimismo, investigaron sobre las políticas públicas dirigidas a la juventud, orientadas a la inserción laboral y la inclusión educativa.

En esta misma línea, recuperamos un trabajo de Acín (1999) sobre valoraciones acerca de la relación educación-trabajo, donde comenta que para los empresarios, en la educación operan tradiciones e inercias que contribuyen a acentuar el desfase estructural existente entre ambos mundos. Algunas de ellas son: planes y programas con tendencias enciclopedistas, desarticulación entre teoría y práctica, desactualización de contenidos y que las escuelas técnicas son más semejantes a otras escuelas secundarias que a las organizaciones productivas.

Para el caso de las pasantías (que es uno de los formatos de PP), Acín (1999) encontró que los docentes tienen una valoración negativa respecto a las prácticas que realizan en las empresas. Los docentes señalan que los empresarios hacen una cierta "utilización" del pasante para actividades secundarias o de apoyo, que no contribuyen directamente a su formación. Además, mencionan que cuando los estudiantes se insertan en la empresa, en algunos casos decrece su rendimiento en el aula, produciéndose abandonos al no poder cumplir con las exigencias horarias en ambos espacios. Finalmente, mencionan la ausencia de seguimiento en las tareas que realizan los pasantes. 
Respecto a los estudiantes, quienes han participado en pasantías las valoran positivamente, consideran que ofrecen la posibilidad de ingreso a un lugar determinado con un perfil semejante a la educación recibida y logran visualizar la aplicabilidad de los contenidos estudiados en la institución educativa.

Otro punto de vista a mencionar es el de sindicalistas entrevistados, quienes hacen un reconocimiento a la formación provista por el sistema educativo y valoran la contratación de mano de obra local por parte de las empresas. No obstante, expresan fuertes críticas tanto a la relación teoría-práctica como a los resultados obtenidos. Este cuestionamiento radica en la falta de práctica en las instituciones educativas, a raíz de una carencia de equipamiento adecuado en tipo, cantidad y calidad, y a su desactualización de contenidos/saberes en función de los requerimientos del mercado laboral. Critican, simultáneamente, los procesos de capacitación en las grandes empresas debido a la ausencia de teoría, al igual que los actuales sistemas de ingreso y capacitación a cargo de agencias privadas.

Algunas conclusiones de esta investigación aportan que las dificultades en la articulación entre educación y trabajo responden a condiciones objetivas y subjetivas. Las primeras incluyen las condiciones de trabajo de los docentes y la insuficiente o inexistente infraestructura y en equipamiento tecnológico en las instituciones educativas. Las segundas tienen que ver con rasgos que han caracterizado a una parte del empresariado argentino, entre ellos: falta de visión a largo plazo, escasa valoración de la capacitación de sus empleados, tendencia a reclutar mano de obra con baja calificación. Por otra parte, los prejuicios que muchos docentes tienen acerca de los empresarios, lo cual está asentado tanto en las características mencionadas como en el desconocimiento mutuo y la ausencia de interacción entre ambos.

En coincidencia con lo encontrado por Acín (1999), Maturo (2018) en su investigación realizada en una escuela secundaria técnica de Córdoba (Argentina), señala que los estudiantes, si bien entienden que la pasantía no siempre puede brindar los resultados esperados, aun así le dan un sentido práctico, que se encuentra ligado a la posibilidad de ingresar a determinada empresa y a su futura efectivización laboral, una vez concluida esta instancia de formación.

Específicamente para el caso de las PP, tanto del sector Madera y Muebles como el de Construcciones, Florez, Luraschi y Butti (2017) señalan, en su trabajo de investigación realizado en el Chaco (Argentina), que los docentes de las escuelas secundarias técnicas comparten la visión de que la realización de las PP tienen limitaciones con respecto a los espacios y modalidades de implementación.

Los jóvenes refieren aprender continuamente y resignificar las acciones formativas en cada situación y de acuerdo con sus proyectos personales de vida, lo que incluye sus experiencias actuales, expectativas y/o posibilidades a futuro. Sin embargo, algunos estudiantes que realizaron las PP también señalan algunos aspectos negativos: la demanda excesiva de tiempo personal 
que los retrasa en otras tareas escolares, lo aprendido no les resulta tan útil ya que no continuarán estudiando carreras afines al sector o no se insertarán laboralmente en el mismo.

En síntesis, existen muy pocos estudios vinculados con las valoraciones de los actores en torno a las PP. En general, los trabajos se refieren a cuestiones prescriptivas, normativas y proyectos de investigación referidos a su implementación en el nivel secundario.

En el proceso de expansión y diversificación de la educación superior no universitaria en Argentina, principalmente surgen dos modalidades de carreras: las destinadas a la formación de docentes y las que apuntan a formar técnicos y profesionales de nivel intermedio.

Deteniéndonos específicamente en la formación técnico-profesional en el nivel superior no universitario, su desarrollo es mucho más reciente que la creación de las carreras docentes, aunque es paralelo a las distintas trasformaciones que estas tuvieron en las últimas décadas. Sin embargo, con pocas excepciones, se trata de programas de estudio nuevos que todavía no están posicionados entre los estudiantes ni entre los empleadores, y cuya efectividad aún se desconoce.

\section{El sector de informática y telecomunicaciones en Argentina}

Según el INET, la definición del sector de Informática y Telecomunicaciones se torna compleja debido a que, por su propia dinámica, presenta la necesidad de actualizar frecuentemente las categorías (actividades) que lo conforman (MEN, CFE, 2010).

La sección de software y servicios informáticos (SSI), junto con la de Telecomunicaciones, conforma el sector de Tecnologías de la Información y la Comunicación (TIC). Este sector es estratégico en la economía de cualquier país globalizado. Existe una relación directa entre la incorporación de TIC y la productividad y competitividad de las empresas.

De acuerdo con estudios internacionales, la cuarta revolución industrial se está desarrollando de la mano de tecnologías disruptivas como la inteligencia artificial, la robótica, la Internet de las cosas, los Big Data, la impresión 3D y los sensores inteligentes.

Si bien el epicentro de esta revolución está fuera de la región, Argentina se ubica entre los 15 países que más comercializa (tanto exportación como importación) los servicios de software a nivel mundial. Pero su representatividad en la producción de hardware es prácticamente nula.

El sector TIC en este país crecerá por encima del Producto Bruto Interno (PIB) (MEN, INET, 2010). Indicador de ello es que entre 2007 y 2016 el número de empresas creció 37\% (Cámara de la Industria Argentina del Software - CESSI, Observatorio Permanente de la Industria del Software y Servicios Informáticos - OPSSI, 2019).

A pesar de este contexto favorable, los perfiles más difíciles de conseguir para las empresas son los especialistas en informática/programación y electrónica, frente a la tendencia a la incorporación de software industriales a lo largo del proceso de diseño, fabricación, control y gestión en los establecimientos productivos. En marzo de 2020 en Argentina, antes del inicio 
del aislamiento social, preventivo y obligatorio (ASPO) producido por la pandemia de COVID-19, las empresas estimaron necesitar un incremento de $14.6 \%$ de su personal $(16,000$ puestos de trabajo) (CESSI, OPSSI, 2020).

La creciente implementación de robots y sistemas de software en el proceso de diseño y desarrollo de productos, en las líneas de producción y montaje, en control de calidad, comunicación, logística, manejo de los inventarios y la posventa, llevan a las empresas a prever que el manejo de herramientas informáticas y la disposición a adaptarse a las nuevas tecnologías serán las habilidades que ganarán mayor valoración entre sus empleados en puestos técnicos (MEN, INET, 2016).

Así, una parte muy importante de los trabajos del futuro -no tan lejano- requerirá conocimientos de informática. Morduchowicz (2009) explica que si en el siglo XV, con la invención de Gutenberg se pasó de la cultura oral a la escrita en el siglo XX, de la cultura de la palabra a la de imagen, en el siglo XXI se ha pasado de la lectura lineal a la percepción simultánea. La revolución tecnológica está trasformando radicalmente nuestras vidas, la manera de comunicarnos, de discutir, de hacer reuniones, de estudiar $y$, también, de producir y consumir.

Pero a esta nueva cultura comunicacional (Castell, 2007) no acceden ni forman parte del mismo modo los diversos sectores socioeconómicos y generacionales de nuestra sociedad. Se observa una gran heterogeneidad entre distintos sectores productivos y segmentos empresariales, así como también entre sectores sociales. Así, por ejemplo, se destaca el rezago de la digitalización en las Pequeñas y Medianas Empresas (Pymes), los sectores de menores ingresos, los habitantes de áreas rurales, las mujeres y las personas de la tercera edad.

Este atravesamiento de la vida contemporanea por las nuevas tecnologías de la información y comunicación, demanda una alfabetización informacional que viene trasformando los procesos de inclusión social de los individuos, lo cual impone el desarrollo de destrezas en tecnologías de la información necesarias para el trabajo y para la vida diaria (Bawden, 2001).

Con esto queremos decir que, más allá del sistema productivo, toda la cultura está atravesada por las nuevas tecnologías y los jóvenes no están exentos de ello. Quienes nacieron en este siglo han conocido desde su infancia un universo mediático extremadamente diversificado.

En síntesis, se trata de un sector complejo signado por: a) la dinámica que le imprime la frecuente actualización tecnológica; b) la obsolescencia de sus productos administrada por la propia industria, por lo que la creación y modificación de actividades profesionales es mucho más frecuente que en otros sectores socioproductivos; $c$ ) lo diverso de las futuras inserciones y trayectorias laborales de los estudiantes, que ponen en tensión la noción de empleo tradicional (Belmes, Rodrigo, 2018).

En esta clave, asumir tal complejidad invita a explicitar el abanico de posibles inscripciones profesionales que pueden involucrar al empleo formal, pero también, entre otros, los emprendimientos personales, el asociativismo, la actividad científico-tecnológica y la docencia (Belmes, Rodrigo, 2018). 


\section{La Tecnicatura Superior en Soporte de Infraestructura de Tecnología de la Información}

En el sector informática, el nivel superior no universitario ofrece dos tecnicaturas:

- Tecnicatura en Soporte de Infraestructura de Tecnología de la Información.

- Tecnicatura en Software.

La primera es a la que se refiere nuestro estudio. El marco de referencia describe el perfil profesional de la siguiente manera:

El Técnico Superior en Soporte de Infraestructura de Tecnología de la Información estará capacitado para implementar, mantener, actualizar, analizar inconvenientes y resolver problemas derivados de la operación de productos de tecnología de la información que cumplen funciones de sistema operativo, administración de almacenamiento, comunicaciones y redes, seguridad, bases de datos, y otros subsistemas, para garantizar la máxima disponibilidad del ambiente operativo de las aplicaciones informáticas de las organizaciones desarrollando las funciones descriptas en el perfil profesional y cumpliendo con los criterios de realización establecidos para las mismas, para lo cual coordinará o complementará su trabajo con especialistas de la misma organización o externos (MEN, CFE, 2010).

Son sus funciones:

- Administrar servidores, software de base, comunicaciones y demás subsistemas, maximizando el aprovechamiento de los recursos y anticipando posibles problemas.

- Administrar redes de comunicación de datos, cableadas o no, asegurando la accesibilidad de los servicios y optimizando los recursos.

- Atender incidentes que afecten la infraestructura de TI, diagnosticar las causas que los originan y resolverlos o coordinar su solución.

- Instalar o remplazar componentes de la infraestructura de TI o adaptarla a nuevas condiciones de servicios externos minimizando riesgos para la seguridad y continuidad del servicio.

- Migrar o convertir sistemas, aplicaciones o datos tratando de minimizar riesgos para la seguridad y continuidad del servicio.

- Entender temas de contingencias y riesgos que puedan afectar a la infraestructura de TI (MEN, CFE, 2010).

En cuanto a las capacidades trasversales que deben poseer y/o desarrollar los técnicos durante su formación, especifica las siguientes: abstracción, razonamiento inferencial, anti- 
cipación, control sobre la calidad integral de sus acciones, trabajo en equipo, comunicación apropiada y actitud de actualización permanente.

Con respecto al área ocupacional, este técnico puede desempeñarse en centros de procesamiento de datos, ya sean de empresas u organizaciones de cualquier tipo, usuarias de tecnología de la información; o empresas que brindan servicios a estas, sin que se requiera su presencia física permanente.

Por último, en relación con el campo de formación de las PP, este marco define que está destinado a posibilitar la integración y contrastación de los saberes construidos en la formación de los otros campos, y garantizar la articulación teoría-práctica en los procesos formativos a través del acercamiento de los estudiantes a situaciones reales de trabajo, que propicie una aproximación progresiva al campo ocupacional hacia el cual se orienta la formación, y poniendo a los estudiantes en contacto con diferentes situaciones y problemáticas que permitan tanto la identificación del objeto de la práctica profesional como la del conjunto de procesos técnicos, tecnológicos, científicos, culturales, sociales y jurídicos, involucrados en la diversidad de situaciones socioculturales y productivas relacionadas con un posible desempeño profesional.

En el diseño curricular, el trayecto de PP se desarrolla a lo largo de los tres años de la carrera, con cinco horas cátedra en el segundo cuatrimestre del primer año, seis u ocho horas en el segundo año y diez horas en el tercero.

\section{Metodología}

El trabajo de campo se realizó en el mes de mayo de 2019, con una muestra intencional de tres carreras técnicas de nivel superior de la provincia de Corrientes (Argentina). Fueron entrevistados: un miembro del polo IT Corrientes; ${ }^{1}$ dos técnicas de la Dirección de Educación Superior; dos técnicos de la Dirección de Educación Técnica del Ministerio de Educación de la Provincia de Corrientes; un rector; dos coordinadores de subsedes; cuatro docentes de PP; tres tutores externos y nueve egresados. Las encuestas fueron aplicadas a 59 estudiantes.

Las entrevistas fueron transcritas y procesadas con el programa Atlas Ti. Se segmentaron frases como unidades de significado, identificando las categorías emergentes e incluyéndolas luego en las categorías principales. Las categorías principales surgieron de los objetivos. En lo que se refiere a los cuestionarios, las preguntas cerradas se procesaron con el paquete estadístico SPSS y las abiertas con el mencionado Atlas Ti.

\section{Descripción de los casos seleccionados}

Como expusimos anteriormente, se seleccionaron tres carreras técnicas de nivel superior, una perteneciente a una institución ubicada en zona rural, otra en zona semiurbana y la última en una zona urbana, con el supuesto de que el territorio incide en múltiples sentidos (tanto en el

1 El Polo IT Corrientes se define como una organización asociativa que reúne empresas del sector de la industria del software y servicios. 
perfil de los estudiantes como en los tipos de prácticas que se pueden configurar según el mercado de trabajo más próximo).

En las tres instituciones encontramos que los estudiantes tienen padres con niveles educativos muy bajos y ocupaciones informales de escaso nivel de calificación, ninguna vinculada con el sector informático. Se reparten casi equitativamente entre varones y mujeres (60 y 40\%, respectivamente). Viven en la localidad donde se encuentra el IS o en localidades aledañas. Entre los motivos de elección de carrera se destacan: la consideración de que le ayudará a conseguir trabajo (28\%) y porque les gusta (24\%). Solo $30 \%$ trabaja regularmente y $11 \%$ realiza trabajos temporales, 4 de ellos (7\%) en tareas del sector TIC.

\section{Resultados y discusión}

\section{Significados}

Respecto a los significados que los distintos actores le otorgan a las PP, en general los técnicos del Ministerio de Educación, los responsables de las instituciones educativas y los profesores las consideran como el eje vertebrador de la carrera, pero que debe ser perfeccionado y jerarquizado. También creen que es necesario trabajar para que tengan un mayor reconocimiento, tanto en los espacios educativos como para los actores e instituciones del mundo del trabajo.

Según refieren, se está iniciando cierto debate acerca de la especificidad de este dispositivo formador en el nivel superior técnico. Para Muller (2002), dentro del ciclo de políticas públicas, un tema se incluye en la fase de problematización o agenda si reúne las siguientes condiciones: que un grupo más o menos organizado defina la situación como problemática o alejada de como debería ser, que sea calificada como parte de la esfera de competencias de las autoridades públicas, y que se espere la intervención de la sociedad política. De las entrevistas realizadas se infiere que la primera de estas condiciones ya está establecida.

La complejidad del tema radica en que se trata de un espacio que incluye componentes pedagógicos -ya que es un espacio curricular-, pero que a la vez excede esta dimensión. Efectivamente, en tanto sitúa al estudiante en el campo profesional y lo conecta con diferentes actividades en contexto, incluye otros componentes de índole sociolaboral. En esta complejidad está el desafío de pensar y diseñar estos espacios de prácticas.

En primer lugar, creo que hay que generar una reconfiguración y que hay que prestarle un poco más de interés en relación, te diría, comparativamente con lo que pasa en la formación docente. En formación docente, se insiste que la práctica es el eje transversal de la formación. Bueno, habría que ver eso también, ya es un cliché a esta altura, pero en el caso de las tecnicaturas no está tan establecido que las PP sean un eje transversal de la formación. Me parece que hay que insistir un poco más. Y creo que tanto la universidad como los institutos, los órganos de gobierno, en este caso Ministerio, direcciones de nivel y demás, tal vez deben empezar a generar instancias de discusión, jornadas, encuentros donde se priorice

Diólopos sobre Educación año 12 | número 22 | enero-junio 2021 | ISSN 2007-2171 
un poco esto, quizá por perfiles de formación, porque no es lo mismo llevar adelante el trayecto de la práctica en esta tecnicatura, objeto de investigación, que por ahí en la tecnicatura en enfermería (Rector del IS3).

En las PP los alumnos pueden aprender mejor, entienden mejor e internalizan la carrera, que no sea muy teórica. Con las PP ven de pronto cómo puede funcionar un drone, por ejemplo, que acá en la localidad todavía no es tan frecuente, y cuál es la aplicación de ese drone, que es transversal a cualquier tipo de actividad; o cómo puede generar una aplicación para utilizarla para determinada finalidad (Coordinador del IS2).

Es muy normal que en una empresa, en una organización, te digan "con experiencia" y ¿cómo vos vas a entrar a trabajar si no tenés experiencia? Entonces las PP son esenciales, son vitales, porque inclusive el alumno puede darse a conocer, al experimentar las cosas afuera puede decidir si quiere o no seguir la carrera (Profesor de PP del IS3).

Según los estudiantes, las PP están orientadas a la consolidación y aplicación de lo aprendido, la comprensión del mundo del trabajo y la formación en otros saberes más vinculados a lo actitudinal, como son las capacidades sociolaborales.

En mi opinión los días de práctica son bastante útiles porque, a diferencia de alguna que otra materia, siento que esta sí pertenece a esta carrera. Además, son más útiles cuando el profesor tiene experiencia laboral, como en mi caso (Estudiante del IS3).

Los egresados resaltan cómo va evolucionando la "confianza" o la capacidad para "desenvolverse" en diferentes situaciones al avanzar en la formación; hay referencias a ir perdiendo "el miedo" a enfrentar ciertos problemas y buscar una solución. Esto no se refiere exclusivamente a competencias técnicas-operativas, sino también a habilidades sociales para enfrentar situaciones de la vida laboral y cotidiana. Probablemente, uno de los efectos más notables de los espacios curriculares que incorporan la dimensión de la PP es el modo en que incrementan la percepción de autoeficacia de la persona. Esto se desarrolla a partir de enfrentar situaciones reales del campo profesional e ir construyendo respuestas sobre la base de los recursos adquiridos en la formación. No es tan solo ganar confianza en uno mismo, sino experimentar la posibilidad de un saber-hacer eficaz para resolver situaciones problemáticas.

Antes de la tecnicatura quizá me daban un teléfono para que le instale una aplicación o lo que sea, y yo,

al desconocer, les decía “iNo, dale a una persona que sepa!” Y ahora como que esta carrera me enseñó 
a nunca decir "No, yo no sé...", a buscar, a tratar de solucionar. Hay cosas que antes necesitamos de un profesional y al día de hoy lo podemos solucionar nosotros y logramos evitar gastos... Las PP me llevaron a animarme cada vez más y a aprender más, también... El día de hoy yo no le temo a quedarme sin trabajo, más allá de que tengo la comodidad de que trabajo en mi lugar, yo con todo esto tengo la seguridad de ir presentarme en una entrevista laboral, tengo la confianza, o sea, no me da miedo a salir a buscar trabajo porque tengo las herramientas... (Egresada del IS1).

\section{Oportunidades}

Esta producción de significaciones -signadas por la valoración positiva y la potencialidad del dispositivo- deben considerarse desde la perspectiva de los roles que ocupan los diferentes actores, enmarcados en sus compromisos institucionales, trayectorias, intereses y expectativas. De allí que al valorar las PP algunos privilegian ciertos aspectos en lugar de otros.

Los estudiantes y graduados tienden a destacar de las PP el potencial que tienen de generar una experiencia formativa y significativa en relación con el mundo del trabajo y, como estudiantes, poder transitar por este espacio. En este sentido, valoran las relaciones con un ambiente laboral: aprender formas de trabajo en una organización, enfrentar problemas reales y concretos del mundo del trabajo, etc.; así como las relaciones sociales: conocer otras personas, ampliar los vínculos, ser conocidos por potenciales empleadores, etc. Algo fundamental que los estudiantes valoran es el bagaje de nuevos conocimientos producidos en contexto (en situaciones reales) y el capital social que propician. Lo cual tiene su sentido considerando sus propias trayectorias formativas personales y sus expectativas a futuro.

Otro aspecto que aparece muy valorado por estudiantes y egresados es la posibilidad que ofrecen las PP de poner en práctica los conocimientos teóricos. Esto amerita un análisis comparativo con la visión de los docentes ya que estos entienden que la dimensión práctica también está en otros espacios curriculares de la formación y que los cursantes ya cuentan con herramientas para desenvolverse en situaciones reales y concretas. La percepción de los estudiantes, por su parte, no suele coincidir con esta apreciación. Desde su perspectiva, las PP son uno de los momentos decisivos en su trayectoria formativa, en el cual son interpelados acerca de la relación teoría-práctica y donde hay una suerte de puesta a prueba de las capacidades desarrolladas en la formación. Sumado a eso, algunos pudieron ascender laboralmente gracias a los conocimientos adquiridos.

Nos da una visión más acabada sobre el mundo laboral y nos brinda las herramientas necesarias para poder desempeñar la profesión de manera correcta. Son realmente útiles ya que al terminar la carrera ya vamos a tener experiencia acerca del mundo laboral en el que nos desempeñaremos (Estudiante del IS3). 
[...] y oportunidades, muchas porque, por ejemplo, el título a mí me sirvió para que me suban mi categoría en el ámbito laboral (Egresada del IS1).

\section{Limitaciones}

Con respecto a las limitaciones, todos señalan en primer lugar el contexto, tanto en lo que se refiere a la poca diversidad de lugares para hacer las prácticas como en cuanto a la distancia (los estudiantes de los parajes o municipios cercanos, en algunos casos no disponen de transporte público o, si existe, no coinciden con los horarios de la institución). Florez, Luraschi y Butti (2017) encontraron resultados similares respecto a las PP vinculadas al sector forestal y al sector construcciones en la región.

Si las PP se realizan en la propia institución, una limitación importante es la falta de equipamiento e insumos, principalmente la insuficiente conectividad. El tema de la falta de conectividad a Internet es un problema provincial. En este sentido, podemos decir que hay esperanzas en que esto se solucione, porque el Estado creó una empresa mixta (con alta participación estatal) que se está ocupando de la conectividad en toda la provincia.

No sé, te podría decir, a nosotros lo que nos limitó muchísimo fue el tema del internet. Acá no hay señal, la velocidad es muy lenta, no tenemos cableado de fibra óptica; así que nos manejábamos con el celular y vos imagínate con el celular estar así [hace un gesto con el teléfono], tenés que estar agrandado y achicando. Y lo que nos faltó también fue práctica, o sea salir a practicar en algunos lugares (Egresada del IS1). Hoy tenemos esperanza. Hay un cambio de gobierno provincial con algo orientado a lo tecnológico. Si yo como empresa quiero hacer un desarrollo, no puedo vender mis productos porque no hay conectividad, entonces yo tengo que mirar hacia otro mercado donde hay más conectividad. El gobierno creó una SAPEM [Sociedad Anónima con Participación Estatal Mayoritaria] que es una empresa mixta, como un modelo ya conocido en otras provincias. Estaría bueno que el Estado esté, que haga usufructo de su red pero que no ponga el pie sobre el sector privado (Presidente del Polo IT).

En segundo lugar, una limitación importante para los responsables institucionales es la falta de normativa jurisdiccional, de la formalización de acuerdos y convenios y el pago del seguro. Respecto a la normativa jurisdiccional, el problema está asociado a la dependencia de la Dirección de Educación Superior, donde la mayoría de las instituciones son de formación docente.

Las oportunidades que hay son buenas, pero se podría mejorar si hubiera más convenios con distintas organizaciones. Las limitaciones justamente son ésas, las de contar con pocas organizaciones a la hora de intentar plasmar lo aprendido en las prácticas (Estudiante del IS3).

Y en el marco de esos convenios, a veces un tema álgido suele ser el del seguro (Técnica de la Dirección de Educación Superior). 
Ahora, en lo que hay un vacío es en la parte de normativa. Nosotros nos regimos por la 1425/14, que es la que nos rige el reglamento orgánico institucional y habla mucho más de la parte docente y no tanto de la técnica. Salió una ampliatoria, no me estoy acordando el número, y aun así falta mucho para la parte de PP. Creo que en el artículo 51 habla sobre PP pero no está reglamentado (Coordinador del IS1).

Las limitaciones del contexto socioproductivo se traducen en las expectativas de los estudiantes respecto al egreso y su futuro laboral. La mayoría considera que no necesariamente se desempeñará en un trabajo relacionado con la carrera.

Una primera cuestión que se remarca son las pocas ofertas de trabajo que tiene el mercado laboral en la actualidad. En este sentido, los encuestados se refieren a pocos o nulos "puestos de trabajo" o "cupos", en directa alusión a una idea de empleabilidad en empresas o en otros organismos del lugar. Se menciona que, en muchas oportunidades, las empresas solo requieren un técnico para cuestiones puntuales, lo que hace que no se plantee como una contratación permanente, sino que se demanden sus servicios ante un problema concreto a resolver.

Es interesante observar que esta característica de escasos puestos de trabajo en el mercado laboral la ubican en el ámbito de sus respectivas localidades. En consecuencia, insisten en la idea de que para conseguir un trabajo -en el que se formó la persona- hay que ir a buscarlo a otra localidad, a otra ciudad o, directamente, a otra provincia. No aparece la idea del teletrabajo ni la del emprendedurismo.

Otro obstáculo que se menciona es la falta de experiencia laboral previa a la hora de conseguir un trabajo. Según los encuestados, esto es algo muy valorado por el empleador para acceder a un puesto laboral.

En esta zona no hay trabajo. En otras zonas por ahí hay que buscar, ya tenés que pensar en dejar tu familia y entonces ya es... no te quiero decir que si yo me voy a la Capital o donde vaya no voy a conseguir, a lo mejor que sí, pero te impiden muchas cosas. Yo pienso que el requisito más importante es que vos tengas mucho conocimiento sobre todo lo que es la parte de tecnología, tener bastante conocimiento sobre eso y tener otro idioma, también, y que uno tenga libertad. Yo, por ejemplo, no me iría al Chaco a buscar un trabajo porque yo ya tengo formada mi familia y como trabajé muchos años tal vez en otra cosa, entonces no voy. Pero una chica joven, si le ofrecen, irá. Y eso es lo que generalmente la empresa busca, no tener limitaciones con sus empleados. Claro y el tema horario, que tenés que irte temprano, volver tarde y tener movilidad, de que por ahí acá es un pueblo, no hay tantas cosas en la tecnología, recién se está armando, después tal vez con los años sí, formen distintos tipos de empresas. Pero ahora, recién se está capacitando el pueblo también con la tecnología. A eso yo me refiero porque, por ejemplo, todas las casas no tienen internet (Egresada del IS1).

El siguiente cuadro sintetiza los resultados descriptos en páginas anteriores.

Diólo@os sobre Educación año 12 | número 22 | enero-junio 2021 | ISSN 2007-2171 
Cuadro 1. Valoraciones de las prácticas profesionalizantes de los distintos actores de la Tecnicatura Superior en Soporte de Infraestructura de Tecnología de la Información de la provincia de Corrientes (Argentina)

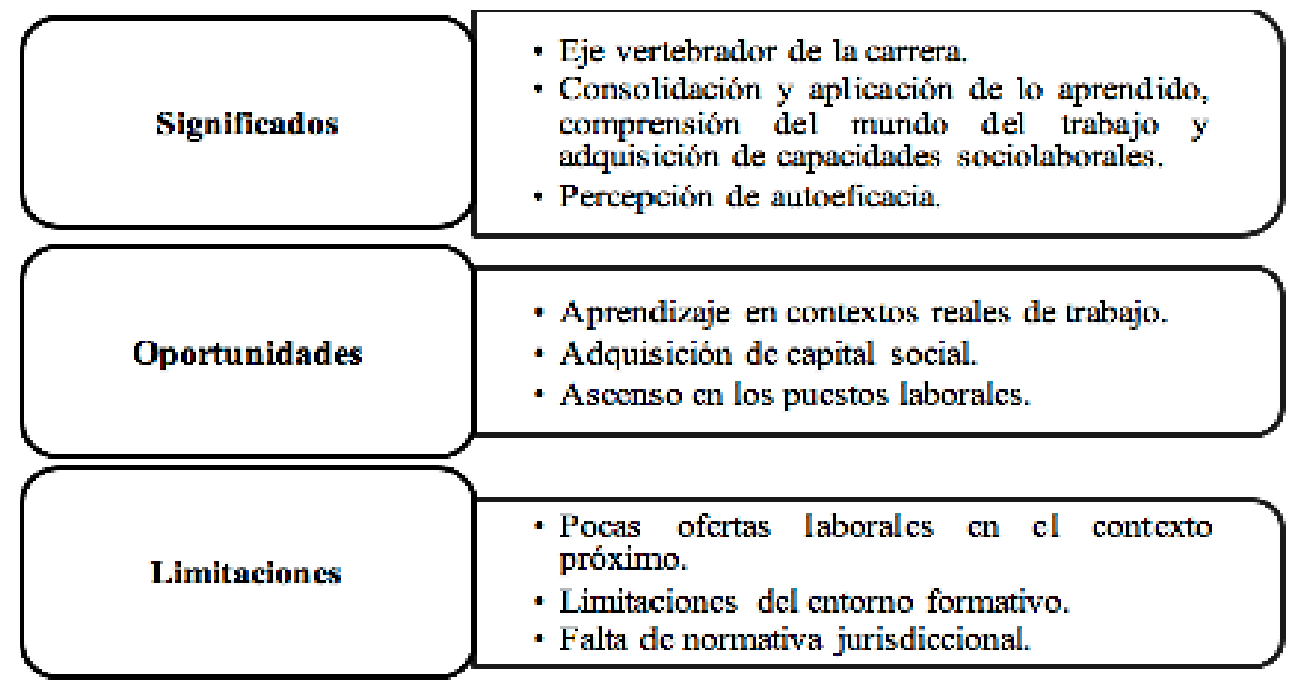

\section{Conclusiones}

En el discurso de nuestros entrevistados amerita ser destacado: la amplia coincidencia en la valoración positiva que hacen de las PP, aunque -claro está- con matices diferentes desde sus posicionamientos e intereses particulares. En este sentido, hay una estimación compartida de que, como dispositivo mediador entre la institución educativa y el mundo laboral, detentan un gran potencial en términos de aportes al proceso formativo de las estudiantes.

Para los técnicos del Ministerio de Educación, los responsables de las instituciones educativas y los profesores, las PP constituyen el eje vertebrador de la carrera. Según los estudiantes y egresados, están orientadas a la consolidación y aplicación de lo aprendido, la comprensión del mundo del trabajo y la formación en otros saberes más vinculados a lo actitudinal, como son las capacidades sociolaborales. Los estudiantes enfatizan la aplicación que hacen de los conocimientos y la importancia del hacer. Visualizan como oportunidades que les brindan las PP los aprendizajes que les sirven tanto en lo personal como para lo laboral, la posibilidad de tener una mejor salida laboral, la adquisición de un capital social, es decir, tener una red de relaciones con otras personas que también pueden aportar a conseguir trabajo; el conocimiento de problemáticas actuales, reales y novedosas del mundo del trabajo.

Con respecto a las limitaciones, todos señalan en primer lugar el contexto en cuanto a la poca diversidad de lugares para hacer las prácticas y en cuanto a la distancia. Y si las PP se realizan en la propia institución, una limitación importante es la falta de equipamiento e insumos, 
principalmente de conectividad. En segundo lugar, para los responsables institucionales, las limitaciones importantes son la falta de normativa jurisdiccional, la falta de la formalización de acuerdos y convenios y el pago del seguro.

Cabe señalar que la valoración positiva de las PP se sostiene a pesar de que las condiciones para su realización no siempre son las óptimas y de que algunas actividades realizadas como PP no necesariamente corresponden al perfil estipulado en la formación profesional. En este sentido, la percepción de que se trata de un espacio que genera una experiencia formativa enriquecedora para sus participantes se sostiene, no obstante las adversidades e inconsistencias que pudieran señalarse en su implementación.

\section{Referencias}

Acín, A. (1999). Mundo del trabajo y educación. Valoraciones acerca de esa relación desde la perspectiva de actores representativos: pequeños y medianos empresarios, docentes de escuelas medias y sindicalistas. Páginas. Revista de la Escuela de Ciencias de la Educación, 1(1). Córdoba: Universidad Nacional de Córdoba.

Bawden, D. (2001). Information and Digital Literacy: A Review of Concepts. Journal of Documentation. Department of Information Science. City University London. Traducción de Piedad Fernández Toledo y José A. Gómez Hernández con permiso del autor.

Belmes, A. (coord.); I. Rodrigo (2018). Hacia un modelo pedagógico de las Prácticas Profesionalizantes en las escuelas técnicas de la Ciudad de Buenos Aires. Reflexiones y aportes a partir de las experiencias de las escuelas con especialidad en Computación. Buenos Aires: Unidad de Evaluación Integral de la Calidad y Equidad Educativa. https://www.buenosaires.gob. ar/sites/gcaba/files/ueicee2018 hacia un modelo pedagogico de las practicas profesionalizantes.pdf

Castells, M. (2007). Communication, Power and Counter-power in the Network Society. International Journal of Communication, 1(1), 29.

CESSI OPISS (2019). Reporte anual sobre el Sector de Software y Servicios Informáticos de la República Argentina. 2018. Buenos Aires: CESSI. http://www.cessi.org.ar/opssi-reportes-949/index. $\underline{\mathrm{html}}$

CESSI OPISS (2020). Sector SSI/OPSSI Coyuntura 2019-2020 / Comisión de estadísticas de CESSI. Buenos Aires: CESSI. https://www.cessi.org.ar/opssi

CFE (2008). Resolución No 47/08. Lineamientos y criterios para la organización institucional y curricular de la educación técnico profesional correspondiente a la educación secundaria y la educación superior. Buenos Aires: Ministerio de Educación de la Nación.

CFE (2010). Marco de referencia correspondiente a la especialidad de "Soporte de infraestructura de tecnología de la información" de nivel superior. Resolución No 107. Anexo II. Buenos Aires: Ministerio de Educación de la Nación.

Diólo pos 
CEPAL (2019). Juventud e inclusión social. Observatorio de Juventud para América Latina y el Caribe. Santiago de Chile: CEPAL.https://dds.cepal.org/juvelac/

DiNIECE (2003). Definiciones básicas para la producción de estadísticas educativas. Buenos Aires: Ministerio de Educación de la Nación. http://www.bnm.me.gov.ar/giga1/documentos/ EL001041.pdf

Do Pico, M. V. (2013). Prácticas Profesionalizantes. Estrategias que vinculan la educación técnica con el mundo del trabajo. Buenos Aires: Asociación Empresaria Argentina.

Dragone, M.; G. Quinzani (2018). Transformaciones en las Prácticas Profesionalizantes, ¿nuevos sentidos en disputa? X Jornadas de Sociología de la Universidad Nacional de La Plata. Ensenada: Universidad Nacional de La Plata. http://www.memoria.fahce.unlp.edu.ar/trab eventos/ev.11476/ev.11476.pdf

Florez, M. A.; M. A. Luraschi; F. Butti (2017). Las prácticas profesionalizantes en la formación de jóvenes que asisten a escuelas técnicas en dos localidades de la provincia del Chaco. El caso de la orientación Madera y Mueble y Construcciones. 13 Congreso Nacional de Estudios del Trabajo. El trabajo en conflicto. Dinámicas y expresiones en el contexto actual. Buenos Aires. https://www.aset.org.ar/2017/ponencias/12 Florez.pdf

Fontaine, G. (2015). El análisis de las políticas públicas. Conceptos, teorías y métodos. Barcelona: Anthropos.

Gallart, M. A.; M. Miranda Oyarzún; C. Peirano; M. P. Sevilla (2003). Tendencias de la educación técnica en América Latina. Estudios de caso en Argentina y Chile. París: UNESCO, Instituto Internacional de Planeamiento de la Educación.

INET (2007). Documento de Prácticas Profesionalizantes. Versión 2. Buenos Aires: Ministerio de Educación de la Nación.

INET (2010). Caracterización y análisis del sector tecnologías de la información. Buenos Aires: Ministerio de Educación de la Nación. http://catalogo.inet.edu.ar/files/pdfs/info sectorial/ informatica-informe-sectorial.pdf

INET (2016). Demanda de capacidades 2020. Análisis de la demanda de capacidades laborales en la Argentina. Buenos Aires: Ministerio de Educación de la Nación. http://www.inet.edu.ar/wpcontent/uploads/2016/06/2016.06.21 Informe Demandas Laborales 2020 vf.pdf

Jacinto, C. (2015). Nuevas lógicas en la formación profesional en Argentina. Redefiniendo lo educativo, lo laboral y lo social. Perfiles Educativos, 37(148), 120-137. https://doi.org/10.1016/j. pe.2015.11.011

Lascoumes, P.; P. Le Galès (2014). Sociología de la acción pública. México: El Colegio de México. Maturo, Y. D. (2018). La escuela técnica y la pasantía en empresas. Aspectos generales sobre su puesta en acto y sus efectos en las expectativas laborales de los alumnos. Praxis Educativa, 22(1): 40-50. 
Ministerio de Educación Corrientes (2011). Resolución No 612. Prácticas Profesionalizantes de la Educación Técnico-Profesional correspondiente al nivel secundario. Corrientes.

Morduchowicz, R. (2009). Cuando la educación en medios es política de Estado. Comunicar. Revista Científica de Educomunicación. 16(32): 131-138.

Muller, P. (2002). Las políticas públicas. Bogotá: Universidad Externado de Colombia.

ONU (2018). Informe de los objetivos de desarrollo sostenible. 2018. Nueva York: ONU.

Pacensa, M. I.; J. Echeverría (2013). Sentidos de la educación y el trabajo en jóvenes. Revisión de aportes conceptuales y empíricos. $V$ Congreso Internacional de Investigación y Práctica Profesional en Psicología, XX Jornadas de Investigación, Noveno Encuentro de Investigadores en Psicología del MERCOSUR. Buenos Aires: Universidad de Buenos Aires. https://www.aacademica.org/000-054/453.pdf

Poder Legislativo Nacional (2005). Ley No 26.058. Ley de Educación Técnico Profesional. Buenos Aires.

SITEAL (2019). Formación TIC y empleo para los Jóvenes: desafíos y posibles cursos de acción. Conversación entre Georgina Vivanco, Néstor Bercovich y María Teresa Lugo. Buenos Aires: SITEAL. http://www.publicaciones.siteal.iipe.unesco.org/eventos-del-siteal/31/formacion-tic-yempleo-para-los-jovenes-desafios-y-posibles-cursos-de-accion 